\title{
INTEGRACIÓN DE LA ÉTICA EN LA GESTIÓN DE LAS PERSONAS Y LOS MEDIOS PARA LOGRAR LA SOSTENIBILIDAD DEL CAMBIO*
}

\author{
Guillermo Pou Munt \\ Centro de Desarrollo de Éticas Aplicadas y \\ PROMOCIÓn DE CAPITAL Social (Bolivia) \\ Ceassrl@entelnet.bo
}

\begin{abstract}
Resumen
Muchas veces, cuando encaramos los temas relacionados con el campo de la ética, tendemos a reducir su significado a las buenas voluntades, abandonando la consideración de la ética como factor clave en la gestión de las organizaciones. Esta omisión es importante, no sólo por la necesidad de tomar decisiones y orientar acciones en función de principios objetivos y contundentes, sino también por los múltiples beneficios que implica el trabajo sostenido y consistente orientado a incluir la ética como una ámbito de gestión, merecedor de tiempo y atención de la gerencia. Para ello es preciso asumir tareas relacionadas con la inclusión de la ética en todos los ámbitos que forman parte de la gestión de las personas dentro de la organización. En este trabajo desarrollamos elementos y criterios sobre ello como un referente para asumir esta temática desde una perspectiva gerencial directamente relacionada con la gestión de las organizaciones y la mejora de su potencial para agregar valor social a la comunidad.
\end{abstract}

Palabras clave: ética, gestión de las personas, gestión de la ética, administración de personal, gestión del personal, gestión del factor humano, gestión de la conducta, competencias morales.

\begin{abstract}
Ethics is a key concept in management, yet it's meaning is reduced to signify just goodwill. Ethics must be an important part of management, as companies should take actions based on objective and conclusive principles. Activities related to ethics should be included in all areas of the company. This is an important omission not only because decisions and actions must be guided by sound and objective principles but also because of the multiple benefits deriving from sustained and consistent work aimed at including ethics as a field of management activities and which therefore deserves management's time and aimed at including ethics in all areas of people management within organizations. This paper will address elements and criteria on how to include ethics in company management from a general perspective and how this can add social value to communities.
\end{abstract}

Key words: ethics, people management, ethics management, personnel administration, personnel management, human factor management, behavioral management, moral responsibilities.

* Una versión anterior de este trabajo fue publicada en los Anales del VIII Congreso de la Asociación Latinoamericana de Ética, Negocios y Economía (Alene), llevado a cabo el 11 y 12 de agosto de 2005 en Valparaíso, Chile. 
$\mathbf{U}$ no de los problemas de las organizaciones que han asumido la labor de trabajar la ética es cómo consolidar los resultados obtenidos por los procesos implementados, ya que la construcción de valores en el ámbito de la cultura organizacional implica el sostenimiento de los mismos en el largo plazo, independientemente de voluntades o personas. Si bien un Código de Ética, que es expresión de valores y principios compartidos en la comunidad organizacional, sirve para este propósito, su utilidad es limitada por diferentes razones y no garantiza la sostenibilidad de los resultados por sí mismo. Por ello, es necesario adecuar las estructuras de gestión con la finalidad de que la ética se integre de forma efectiva y permanente en la organización y sus prácticas.

El presente artículo se orienta al análisis de estos temas y propone, específicamente en lo relacionado a la gestión de las personas ${ }^{1}$, medidas que deben ser asumidas con vistas a consolidar la sostenibilidad del cambio a largo plazo, e integrar los valores y principios promovidos a la cultura de la organización.

La construcción de medios para la integración de la ética a la organización, implica el desarrollo de dos factores claves de la estructura organizativa. Por una parte, aquel que se relaciona con la estructu-

1. La «Gestión de las Personas» hace referencia a lo que tradicionalmente se conoce como «Gestión de Recursos Humanos». Este cambio en la terminología se debe a que el tratamiento de las personas como un recurso más de la organización reduce su condición a un simple objeto semejante a cualquier otro activo, lo cual es cuestionable desde el punto de vista ético. Para evitar ello se modifica el término empleado, aunque este sea de uso muy común en el ámbito de la gestión de las organizaciones. ra de gestión de las personas y, por otra, el que se refiere específicamente a la gestión de la conducta, una variable que debe ser incorporada formalmente en el espectro de la gerencia desde sus dos vertientes, la normativa y, la más importante, la consolidación de una infraestructura ética, que a partir de su especificidad garantice el mantenimiento y sostenibilidad en el largo plazo de una visión basada en valores y principios.

Respecto a la gestión de las personas, proponemos que las actuales políticas son insuficientes cuando se trata de darle a la organización una perspectiva ética, que priorice la promoción de valores morales como elemento clave en el conjunto de los valores organizacionales.

Un entorno en el cual los valores no sean tenidos en cuenta en los procesos de reclutamiento y selección, la inducción no considere la necesaria formación inicial en competencias morales, la evaluación no asuma la buena conducta como referente ni la equidad como política, y la capacitación no se oriente al desarrollo de las competencias morales como una de sus preocupaciones, no se halla en condiciones de garantizar la integración de la ética en las estructuras de gestión ni, menos, su sostenibilidad como paradigma gerencial a partir de su inserción en la cultura organizacional.

Por otra parte, se debe contemplar la construcción de una infraestructura ética, que se sustente en la conciencia, por parte de la organización, de la necesidad de establecer procesos relacionados con la promoción del imperativo ético, que requiere de medios y estructura especializada. Todo ello con la finalidad no sólo de consolidar competencias, sino de pro- 
mover una visión de sostenibilidad de valores, en un entorno en el cual la rotación de personal y el cambio de las condiciones del ambiente externo afectan de forma real la clave de costumbres, ritos y valores compartidos en la organización.

La necesidad de complementar el espectro de instrumentos con un adecuado entorno normativo no debe ser desdeñada. Sin embargo, no debe perderse de vista que la finalidad de esta instancia es la de contar con los medios para tratar la inconducta de forma severa y, en determinados momentos, poder retirar de la organización a aquellos miembros que muestren, a través de continuas malas prácticas -con los consiguientes efectos negativos para la organización-, su rechazo e inconformidad con la clave de valores y principios compartidos.

La infraestructura ética, los ajustes en la política de gestión de las personas y el establecimiento de un entorno normativo razonable son los elementos que permiten consolidar en la organización la base de instrumental de gestión orientada a afianzar la ética como referente para la toma de decisiones, generando aceptación hacia la conducta consecuente con valores y principios, y rechazo hacia la mala práctica.

El entorno normativo, que se diferencia claramente de la instancia ética, se constituye en un instrumento complementario para administrar situaciones específicas inadecuadas, en la medida en que involucran individuos que no asimilan la perspectiva de la conducta basada en convicciones y requieren, por lo tanto, de una intervención drástica para reducir los efectos negativos que pueden generar al deteriorar el clima organizacional.

\section{Los componentes de la gestión de las personas}

En los últimos años se ha consolidado una corriente, a la cual nos adscribimos, que considera el empleo del término «Recursos Humanos» como una referencia reduccionista del rol de las personas en las organizaciones, y que busca establecer la necesidad de «capitalizar» o activar a aquellas (en el sentido de convertirlas en un activo). Si bien los autores que emplean esta referencia pueden estar alejados conceptualmente de esta idea, es necesario replantear el rol de las personas en las organizaciones, lo cual parte de emplear términos adecuados relacionados con los ámbitos de gestión que se aplican a su actuación, motivación y desempeño. En este contexto, se emplea el término de «Gestión de las Personas» como una expresión semántica más apropiada para referirnos a este ámbito de la gestión en todo tipo de organizaciones.

El concepto de gestión de las personas engloba varios criterios relacionados con las variables básicas de administración del personal, aquellas que tradicionalmente se ubican en el ámbito de la gestión de los recursos humanos y que identificaremos como variables de gestión de personal, gestión del factor humano y gestión de la conducta. Cada uno de estos componentes involucra un ámbito de la gestión del factor clave de la organización: las personas.

A continuación delimitaremos los alcances de cada componente.

Administración de personal. Se refiere a las variables administrativas relacionadas con los manuales de funciones, asignación de tareas, responsabilidades y 
autoridad, manuales de cargos, administración salarial, administración de los factores higiénicos del trabajo y otros similares.

Gestión del personal. Se relaciona con los procesos de reclutamiento, selección, contratación, inducción, evaluación y capacitación del personal.

Gestión del factor humano. Es el ámbito del clima organizacional, la gestión del talento dentro de la organización, la satisfacción en el trabajo, la gestión de los factores no higiénicos y otros elementos relacionados.

Gestión de la conducta. Se relaciona específicamente con la promoción y seguimiento de conductas desde la perspectiva ética o con la observación de las mismas desde la perspectiva normativa. En general, se trata de establecer cierto tipo de estándares de comportamiento, considerando las diferencias y potencial distinto de resultados obtenibles desde ambas perspectivas.

En las páginas siguientes desarrollaremos las implicancias de incorporar de forma efectiva el imperativo ético en cada uno de estos componentes, a fin de consolidar un programa de «integridad corporativa» que esté en condiciones de generar un impacto efectivo en el ámbito de la cultura de la organización. De este modo se garantizará la persistencia de ciertos valores y principios en el largo plazo, independientemente de la presencia de determinadas personas o de la voluntad de las gerencias.

\section{Modificación de los componentes con una visión ética}

Para consolidar su proceso de promoción de la ética, la organización deberá intervenir en cada componente del área de gestión de las personas e implementar políticas, procedimientos e instrumentos que permitan garantizar la integración del nuevo paradigma dentro del conjunto de criterios empleados habitualmente en la gestión de la organización. Con esta finalidad se deben desarrollar acciones específicas, que describimos en detalle a continuación.

\section{1. Ámbito de la administración de personal}

El ámbito de la administración del personal comprende las variables básicas de gestión relacionadas con:

- La asignación de funciones y responsabilidades.

- La delimitación del ámbito de autoridad.

- La definición de la carga de trabajo.

- La gestión del salario.

- $\mathrm{El}$ acceso a beneficios.

La gerencia debe asumir que todos estos instrumentos son un medio para contribuir a la satisfacción del personal en el desempeño de sus funciones, por lo cual deben ser administrados bajo criterios de equidad, incorporando políticas que reflejen en la práctica el valor de la gestión de la organización. Entre ellas:

- La asignación de funciones y responsabilidades debe guardar relación con las competencias de las personas, sus capacidades y condiciones para asumir las mismas, de forma que exista 
una verdadera posibilidad de generar resultados y, por ende, alcanzar satisfacción por este medio.

- El ámbito de autoridad debe ser provisto considerando lo que se demanda de la persona a partir de las funciones y responsabilidades otorgadas con el cargo, de forma que la capacidad de decisión no opere como un freno para la consecución de los objetivos que le son establecidos.

- La carga de trabajo debe considerar que la vida de la persona comprende dimensiones distintas al entorno laboral, por lo tanto, la organización debe otorgar a sus trabajadores el tiempo suficiente para que puedan dedicarse a las actividades que les permitan su autorrealización.

- El salario debe ser administrado con equidad, buscando establecer mínimos que garanticen el acceso a una vida digna y a condiciones por medio de las cuales, a partir del esfuerzo propio, el individuo mejore su calidad de vida y la de su familia. Es necesario considerar que el salario debe mantener proporcionalidad con las funciones, responsabilidades, autoridad y carga de trabajo asignadas a la persona.

- El acceso a beneficios implica considerar la necesidad de que el individuo tenga la posibilidad de recibir beneficios adicionales que complementen su ingreso o le generen bienestar, más allá del salario. En este rubro se encuentran los seguros de salud, las condiciones laborales, la seguridad industrial, los gastos de representación y otros elementos que deben asociarse al mérito y al logro de objetivos.
Como puede verse, intervenir en las variables básicas significa iniciar la construcción de la ética en la organización, pues no es posible demandar buena práctica si no se parte de la incorporación de la misma en la actitud y la calidad de la relación que la organización mantiene con sus miembros.

\subsection{El ámbito de la gestión de personal}

Esta variable, central en la construcción de un entorno promotor de la ética, permite a la organización enriquecerse con la incorporación y retención de personas probas, como un elemento generador de condiciones para la consecución de los objetivos organizacionales. Desde esta perspectiva se debe considerar actuar sobre los siguientes elementos:

- Los procesos de reclutamiento y selección.

- Las políticas de inducción del personal.

- Los instrumentos y políticas de evaluación del desempeño.

- Las políticas de capacitación.

Las intervenciones que se debe considerar en este ámbito se detallan a continuación.

- En lo referente a los procesos de reclutamiento y selección, se debe considerar la tendencia ética de las personas. Aunque no es posible establecer cómo es cada individuo desde una perspectiva moral, sí es posible apreciar sus tendencias y predisposiciones, las mismas que permiten darse cuenta si la persona se ajusta o no a la matriz ética que la organización promueve como referente de conducta y toma de 
decisiones. Los procesos subjetivos de selección de pares que habitualmente se aplican deben evolucionar a mecanismos objetivos de búsqueda e identificación de tendencias, predisposición a valores y presencia de competencias humanas, como factores centrales en la selección del nuevo personal.

- Las políticas de inducción del personal nuevo, deben ir más allá de la simple presentación, de tal manera que la persona que recién se incorpora a la organización, además de la lectura del Código de Ética, sea provista de una adecuada capacitación introductoria en el desarrollo de competencias morales. También se deberán generar las condiciones para que el individuo tome contacto con el ambiente de la organización y pueda percibir de primera mano el clima y los valores compartidos. Para ello se puede aplicar programas de compañeros de inducción, grupos de asimilación o programas de intercambio de experiencias.

- Las políticas e instrumentos de evaluación del desempeño deben evolucionar en dos direcciones. Por una lado, deben incorporar instrumentos y mecanismos que garanticen la equidad en la evaluación del desempeño, lo cual implica eliminar los riesgos inherentes a una evaluación vertical, lo mismo que el sesgo de la sola evaluación de los resultados alcanzados: Debe incorporarse mecanismos que permitan establecer desde diferentes perspectivas cómo piensan los clientes internos de la labor desempeñada, a lo cual se debe añadir un programa de mejora continua y desarrollo de competencias profesionales. Por otro lado, se debe buscar activamente que la conducta se constituya en referente central del desempeño y de la posibilidad de acceso a ascensos, permanencia y otras condiciones laborales. Con este fin, se debe contar con bases de datos que registren las actuaciones de los miembros de la organización, guardando el estricto respeto a los derechos de las personas.

- Las políticas de capacitación deben ser integrales y centrarse en el desarrollo de competencias, tanto profesionales como humanas, con la finalidad de mejorar la calidad de la personas y permitirles un mejor desempeño en ambos aspectos. En este ámbito actúan los programas de capacitación continua en competencias morales, cuya finalidad es dotar al individuo de los instrumentos que le permitan superarse como persona, a partir de su propia experiencia, y desarrollar sus capacidades de razonamiento moral, para ser aplicadas en los procesos de toma de decisiones.

La gestión de las variables relacionadas con el personal introduce un ámbito de desarrollo del entorno organizacional en el cual la persona es un objetivo central de las políticas de la gerencia. Con ella se busca generar las condiciones para la sostenibilidad de los valores morales de la organización a partir de la promoción de los mismos, partiendo de la introducción de la equidad y el desarrollo de competencias humanas, como factores clave en la gestión del recurso humano. 


\subsection{El ámbito de la gestión del factor humano}

La necesidad de diferenciar la administración y la gestión del personal, por un lado, del factor humano, por otro, parte de la consideración de que los elementos incluidos en uno y otro ámbito, si bien se dirigen al mismo objeto, lo asumen de diferente manera.

Cuando hacemos referencia al factor humano, estamos centrándonos en ese conjunto de variables que a pesar de poder ser gestionadas no son predecibles en cuanto a sus resultados, ya que introducen componentes que van más allá de la satisfacción en el trabajo. La idea es llegar a consolidar como una preocupación de la gerencia, la necesidad de que los miembros de la organización se sientan felices a partir de la actividad que desarrollan. En este ámbito se debe considerar elementos tales como:

- La gestión del clima organizacional.

- La promoción de valores y principios.

- La generación de condiciones para una vida feliz dentro de la organización.

Aquí tratamos sobre los nuevos roles de la organización respecto a sus miembros, en lo que concierne a la preocupación por la satisfacción del personal más allá del trabajo. En primer lugar, se busca generar condiciones para que las personas se sientan felices trabajando. Esto implica que el impacto de su labor sea percibido como un aporte real al bien común, es decir, que se extienda más allá del ámbito de la organización, lo cual mejorará la percepción de la relevancia de la labor del individuo para la comunidad. Desde esta perspectiva se deben generar condiciones para trabajar bajo las siguientes directrices.
- Se debe buscar fortalecer un clima organizacional basado en valores compartidos en la organización, de los cuales se deriven principios morales claros que sean tomados como referente para las relaciones laborales. Todo ello permitirá configurar el efecto confianza -con todos los beneficios que ello trae para la organización y sus miembros- establecido en términos de mejores condiciones de trabajo, ambiente propicio al perfeccionamiento continuo, alineación con los objetivos institucionales, reducción de los costos de control y de la carga de coordinación. Todo ello constituirá un entorno favorable, en el cual las personas desearán trabajar.

- La promoción de valores y principios se hará bajo un enfoque orientado al afianzamiento de los mismos en la cultura de la organización, lo que significa consolidar estos referentes como un elemento central de gestión.

- Finalmente, encontramos que la preocupación por una vida feliz dentro de la organización ciertamente no se relaciona con el relajamiento de las exigencias o de los objetivos. Por el contrario, se trata de promover el logro de las metas organizacionales, pero a partir de la identificación efectiva de los trabajadores con las mismas.

Se debe generar las condiciones para que la persona desarrolle un sentido de lealtad basado en valores y principios, que se traduzca en un sentimiento de bienestar relacionado con la pertenencia a la organización y a la posibilidad de aportar, por su intermedio, al bienestar de la comunidad. 
Como puede verse, los elementos relacionados con el factor humano son clave para establecer un programa de ética efectivo, pues la conducta se promueve por convicción y no por obligación, otro elemento que aporta a la felicidad de las personas en la medida en que se saben libres.

\subsection{El ámbito de la gestión de la conducta}

Las acciones de la organización orientadas a la promoción de valores deberán tener un resultado práctico en la conducta de las personas. Como se indicó antes, existen dos vertientes que deben ser trabajadas poniendo el acento en la perspectiva ética antes que en la normativa. Para ello debemos construir una infraestructura ética, es decir, un conjunto de medios especializados para trabajar este ámbito con eficiencia, complementados con todo el espectro de elementos que ya han sido descritos en cuanto a las actuaciones que se espera desarrollar. Cuando hacemos referencia a la infraestructura ética estamos hablando de instrumentos tales como:

- El código de ética institucional.

- El comité de ética.

- La unidad de promoción de la ética institucional.

- El sistema de gestión de la ética.

- Círculos de ética.

A continuación describiremos la naturaleza de cada uno de estos instrumentos.

- El código de ética es un instrumento poderoso para la consolidación de valores en la organización cuando se aleja de forma estructural de la visión normativa, que debe estar circunscrita al ámbito de los regímenes disciplinarios. Este documento cobra relevancia cuando es construido sobre la base de valores compartidos, en función de principios que son expresados por los propios funcionarios como parte de un proyecto común con el cual se comprometen.

Además, para consolidarse como referente para la toma de decisiones, el código de ética debe ser construido de forma participativa, en la medida en que expone políticas que son útiles a los miembros de la organización para orientar sus conductas y establecer aquello que es correcto, en presencia de parámetros objetivos.

No debe perderse de vista que lo relevante son los procesos que rodean la construcción e implementación efectiva del código de ética y los compromisos que consolida y permite formalizar. Debe evitarse dar al simple papel un valor que no posee.

El rol de los comités de ética debe ser fortalecido como una instancia coadyuvante de las actividades de promoción de la ética. La tendencia a considerar que se trata de un tribunal destinado a la sanción es errónea y está muy extendida, ya que la ética debe concentrarse en el ámbito del «ser y del deber ser», lo cual se aleja de la simple sanción como medio para la promoción de valores.

La construcción de convicciones se centra en el desarrollo de competencias morales, que se traducen luego en principios asumidos como compromiso por las personas. El comité de ética juega un rol determinante cuando orienta sus funciones hacia la promoción de la capacitación, la coordinación de las acciones de promoción de la ética, la atención de consultas 
o dudas antes de la acción; así como a la solución de situaciones de conflicto y controversia relacionadas con su aplicación, la orientación sobre las decisiones de la gerencia y del personal en general, y la protección a las personas que desarrollan una conducta genuina ante la represalia y la acción interesada.

La función de sancionar es accesoria y debe ser considerada como algo secundario. Las resoluciones del comité de ética, si bien requieren mucha referencia procedimental para su adecuada implementación, deben tener el carácter de una sanción moral que busca el remordimiento y el reproche, mientras que la acción punitiva debe ser derivada a las instancias disciplinarias por los conductos correspondientes. Los temas de las faltas son tratados en el comité con la finalidad de promover la discusión de temas que requieren ser fortalecidos en el ámbito de la organización, como parte de un proceso de mejora continua. La sanción, desde la perspectiva ética, entonces, debe asumir su verdadera dimensión secundaria, derivando la acción tipificadora y sancionadora a las instancias disciplinarias.

- La unidad de promoción de la ética institucional es una instancia de gestión formal que vela por la implementación efectiva de las políticas correspondientes y por el sostenimiento de los procesos relacionados con la consolidación de valores y principios en la organización.

Esta instancia es necesaria para garantizar la sostenibilidad y la inserción del tema como elemento de la gestión. En función del tamaño de la organización, puede ser asumida como una instancia específica o como una función den- tro del ámbito de la gerencia encargada de la gestión del factor humano.

Entre otras tareas, asume la función de coordinar la operación del comité de ética; recibir, procesar y derivar las denuncias a las instancias disciplinarias; coordinar la capacitación especializada; y, en general, promover que las políticas relacionadas con la ética sean efectivamente implementadas y operadas.

- El sistema de gestión de la ética involucra un conjunto de partes que se relacionan entre sí en busca del objetivo central. Esto implica la interacción de las instancias de administración de personal, gestión de personal, gestión del factor humano y la operación de la propia infraestructura ética.

El sistema retroalimenta de forma activa la construcción ética en la organización, a partir de procesos que involucran a la totalidad de los componentes de la estructura organizacional, como objeto de interacción y fuente de consolidación de valores.

- Los círculos de ética son espacios formales donde los empleados, en la informalidad de sus relaciones, encuentran una alternativa para la discusión sobre el diario vivir en la organización. Allí, tratan los problemas que alejan a los miembros de la institución de la efectiva vivencia de los valores y principios que son promovidos.

La continua identificación de debilidades y obstáculos a partir de estos espacios, también permite la identificación de soluciones que van a fortalecer los medios para la efectiva implementación de la ética como imperativo de acción. 
La necesidad de un régimen disciplinario no debe ser desdeñada, pero su implementación debe considerar lo siguiente:

a) El régimen disciplinario es un ámbito distinto al de la instancia ética; no la sustituye, es más bien un complemento en la gestión de la conducta.

b) El régimen disciplinario se sustenta en la clara tipificación de las faltas; puede ser promovido desde la instancia ética con la finalidad de desarrollarse en función de las malas prácticas que se desea evitar.

c) La estructura de punición, es decir, de aplicación de sanciones, debe ser severa para operar como elemento de disuasión. Requiere, además, de voluntad política para su aplicación, lo cual implica la provisión de fondos para la operación de los controles.

d) El régimen disciplinario centra su actuación en el grupo de miembros de la organización que no están dispuestos a asumir una conducta comprometida con valores y principios. Estas personas deben ser disuadidas o, de lo contrario, retiradas de la estructura de la organización para evitar el potencial daño que representan para la misma y para la sociedad.

e) Se deben establecer los procedimientos que permitan la aplicación del debido proceso cuando se administra la justicia administrativa y cuando se pasa de esta a las instancias judiciales civiles o penales.

Si bien se complementan, la instancia ética y el régimen disciplinario o normativo son tan diferentes como el agua y el aceite. Pueden coexistir, pero el pretender mezclarlos genera serios problemas en el ámbito de la gestión de la conducta. Por ello, debe mantenerse clara conciencia de que se trata de ámbitos y enfoques distintos que confluyen de forma diferente en el objeto de la gestión de la conducta y que poseen objetivos distintos en lo referente al desarrollo moral de las personas.

\section{La integración de los componentes del sistema}

La siguiente figura muestra la interacción de todos los componentes del sistema de gestión de la ética institucional y los canales a través de los cuales consolidan sus efectos en la gestión de la organización.

Las relaciones centrales se construyen en torno al sistema de gestión de la ética, que se conforma a partir de la interacción de las políticas e instrumentos ubicados en los cuatro ámbitos que lo componen. De forma complementaria opera el régimen disciplinario que pretende consolidar la conducta desde una perspectiva diferente.

El resultado de la operación del sistema a partir de la vigencia de la infraestructura ética, es decir, de los medios específicos para la gestión de la ética, se traduce en la consolidación de valores y principios, los mismos que se constituyen en componentes clave de la cultura organizacional.

Por esta vertiente se promueve la actuación por convicción que se estima tiene efecto directo sobre un universo que va del $60 \%$ al $70 \%$ del total del personal. El restante $20 \%$ o $30 \%$ es controlado por medio de la disuasión, ya que se trata de personas que no se adscriben a los valores morales promovidos por la organización. 


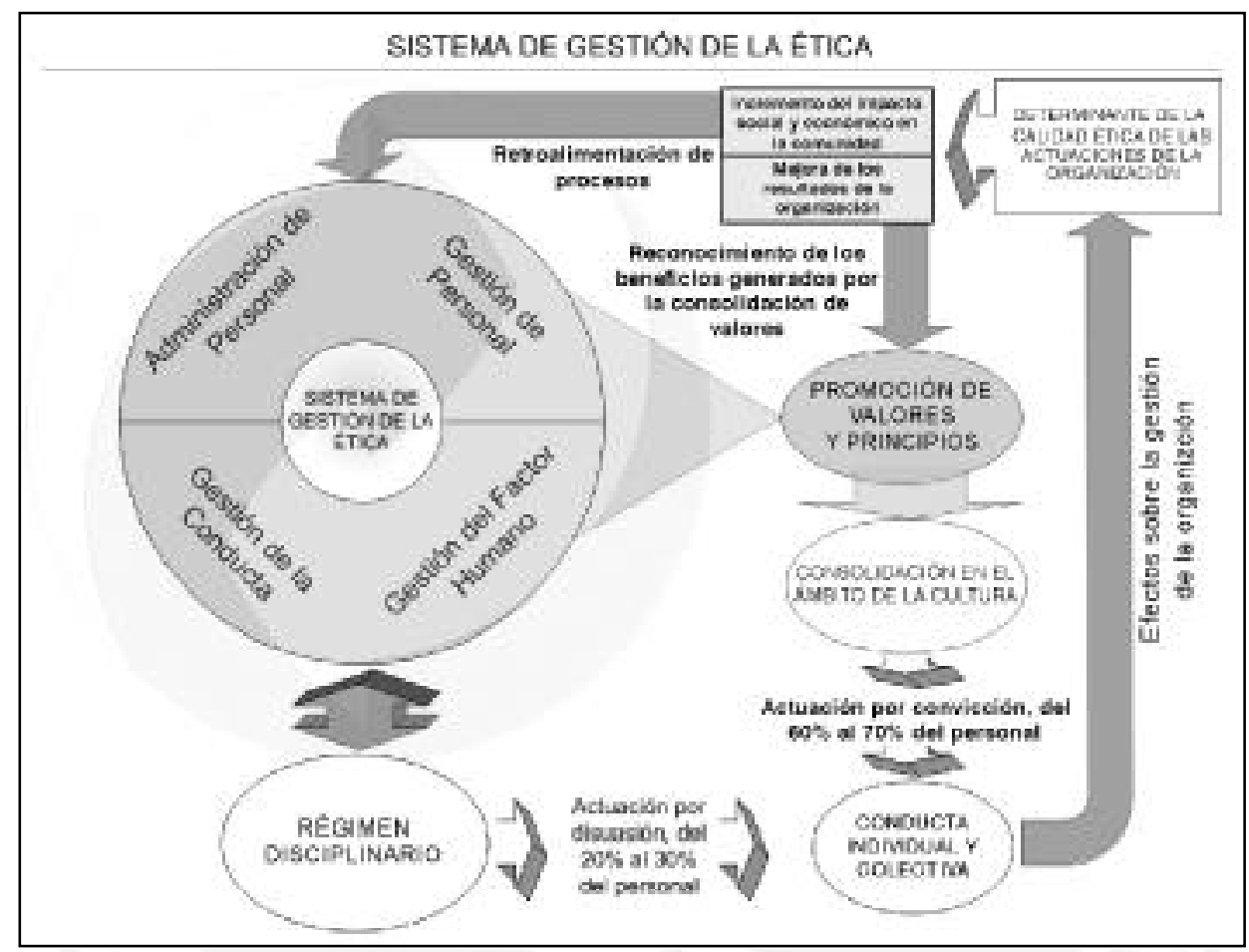

El efecto final de la conducta promovida se traduce en la buena práctica, que se inscribe en la calidad de todas las actuaciones de la organización y, de este modo, mejora el impacto de esta en la comunidad y eleva el potencial de generación de resultados. Ambos elementos justifican la operación del sistema de gestión de la ética y los ajustes que mejoran su actuación, al mismo tiempo que promueven activamente el reconocimiento de los valores y principios compartidos en la organización, como base para la construcción de la integridad institucional.

Puede apreciarse entonces, claramente, que la gestión de la ética posee el potencial para la consolidación de resultados efectivos en el ámbito de la actuación de la organización. Ello justifica plenamente su incorporación como una de las variables de gestión de mayor importancia, junto a la planificación, organización, dirección y control.

\section{Conclusiones}

El presente trabajo ha presentado como un ámbito estructurado de gestión lo que inicialmente era un conjunto de suposiciones e intuiciones. Podemos señalar ahora las siguientes conclusiones:

1) La alternativa de invertir recursos, tiempo y medios en el ámbito de la gestión de la ética promete, a partir del compromiso con valores clave, la consolidación de efectos positivos en la comunidad organizacional y resultados que complementan los beneficios para la organización. 
2) La ética incorporada como un elemento central de la gestión, constituye el medio para consolidar la ciudadanía organizacional, como paso previo a la construcción de programas de responsabilidad social.

3) Las decisiones inspiradas desde una perspectiva ética incorporarán criterios que maximizarán los resultados de la organización y la aceptación de la misma en la sociedad.

4) El impacto positivo en términos de reducción de costos de control y coordinación, además de un clima organizacional estimulante y de alto potencial, significa mejores condiciones de vida dentro de la organización.
5) La aceptación de este enfoque por parte de los miembros de la organización es crucial, pues genera una real participación en los procesos, al mismo tiempo que permite una visión equitativa de la relación entre la organización y sus componentes. Todo ello origina desempeños superiores, al reafirmarse el sentimiento de pertenencia a partir del incremento de la satisfacción de las personas en el trabajo.

Considerar la ética como una cuestión de gestión implica asumir la responsabilidad de construir un futuro mejor para las personas que forman parte de la organización, para la propia organización y para la sociedad en general.

\section{Referencias bibliográficas}

ABRAVANEL, Harry et ál. 1992. Cultura organizacional. Santa Fe de Bogotá: Legis.

BROWN, Marvin. 2001. La ética en la empresa: estrategias para la toma de decisiones. Barcelona: Paidos.

FREDERICK, Robert. 2001. La ética en los negocios. México D. F.: Oxford University Press.

JERICÓ, Pilar. 2001. La gestión del talento: del profesional con talento al talento organizativo. Madrid: Prentice Hall-Financial Times.

OLTRA CLIMENT, Vicente. 1995. Instalarse en el cambio: competitividad y ética. Madrid: CDN Ciencias de la Dirección.

PALADINO, Marcelo. 2004. La responsabilidad de la empresa en la sociedad: cons- truyendo la sociedad desde la tarea directiva. Buenos Aires: Ariel.

PÉREZ LÓPEZ, Juan Antonio. 1999. Fundamentos de la dirección de empresas. Madrid: Rialp.

SCHMIDT, Eduardo. 2003. Ética y negocios para América Latina. $3^{\mathrm{a}}$ ed. Córdova: Universidad Católica de Córdova.

SISON, Alejo; FONTRODONA, Joan y VELAZ, Iñaqui. 2002. Tras la euforia: guía ético para directivos en la nueva economía. Madrid: Prentice Hall-Financial Times.

VELÁSQUEZ, Manuel. 2000. Ética en los negocios: conceptos y casos. México D. F.: Pearson Educación. 